\title{
The comparison of topmost radio occultation electron densities with in-situ ion densities from FORMOSAT-7/COSMIC-2
}

\author{
Chia-Hung Chen ${ }^{1, *}$, Ho-Fang Tsai ${ }^{1}$, Li-Yuan Wang $^{1}$, Chien-Hung Charles Lin ${ }^{1}$, Jann-Yenq Liu ${ }^{2,3}$, and \\ Wen-Hao $\mathrm{Yeh}^{4}$ \\ ${ }^{1}$ Department of Earth Sciences, National Cheng Kung University, Tainan City, Taiwan \\ ${ }^{2}$ Institute of Space Science, National Central University, Taoyuan City, Taiwan \\ ${ }^{3}$ Center for Space and Remote Sensing Research, National Central University, Taoyuan City, Taiwan \\ ${ }^{4}$ National Space Organization, Hsinchu City, Taiwan
}

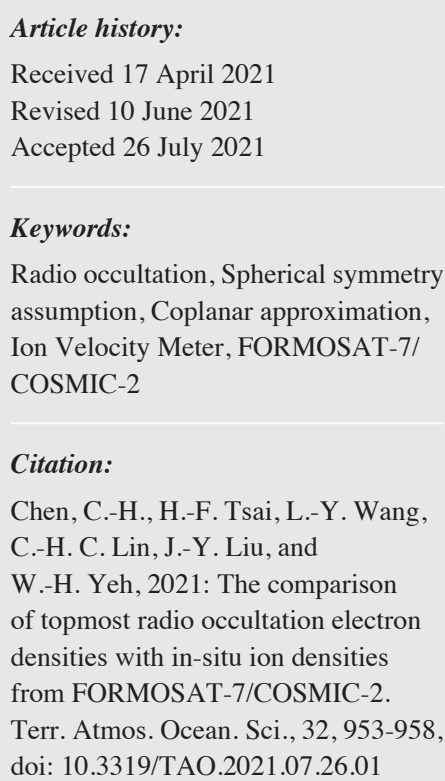

\begin{abstract}
The ionospheric radio occultation (RO) inversion is a powerful tool in retrieving the global electron density profiles (EDPs) remotely by using the time delay of the signals received by Low Earth Orbit (LEO) satellites from the GPS and other GNSS satellites based on the spherical symmetry assumptions and the coplanar approximation. However, these assumptions may cause the inaccuracy in the electron density retrieval. In this study, for the first time, we present an ionospheric electron density comparison between the estimated topmost electron density profiles from the FORMOSAT-7/COSMIC-2 (F7/C2) RO and the co-located in-situ ion densities obtained from the Ion Velocity Meter (IVM) onboard the F7/C2 satellites and then further quantitatively evaluate the impacts of the abovementioned Abel inversion assumptions on the topside ionospheric electron density. Results showed the RO topmost electron density is overall in good agreement with the IVM in-situ ion density but is slightly underestimation. Furthermore, the dihedral angle of the LEO and the occultation plane is also highlighted the importance of the coplanar approximation in the Abel inversion.
\end{abstract}

\section{INTRODUCTION}

The six-satellite FORMOSAT-3/Constellation Observing System for Meteorology, Ionosphere and Climate (FORMOSAT-3/COSMIC, or F3/C in short) launched in April 2006 and provided 1000 - 2500 ionospheric electron density vertical profiles per day through the Global Positioning System (GPS) radio occultation (RO) technique. These abounding observations are extensively employed in the global ionospheric research (e.g., Luan et al. 2008; Lin et al. 2010) and the ionospheric nowcast/forecast system (e.g., Lee et al. 2012; Lin et al. 2017).

The accuracy of the ionospheric peak density $(\mathrm{NmF} 2)$ and the peak height $(\mathrm{hmF} 2)$ from the GPS RO have been reported by comparing with the ground-based ionosonde

\footnotetext{
* Corresponding author

E-mail:koichi0925@gmail.com
}

and incoherent scatter (IS) radar (Lei et al. 2007; Kelley et al. 2009), and the space-based CHAllenging Minisatellite Payload (CHAMP) (Yue et al. 2011; Pedatella et al. 2015), showing the root-mean-square error is about 10 to $20 \%$. This error is mainly caused from the assumptions of the Abel inversion when deriving the electron density profile (EDP), including the topmost electron density estimation and the coplanar approximation. Using the in-situ observations, around 400 to $800 \mathrm{~km}$ altitude, from the Communications/Navigation Outage Forecasting System (C/NOFS) satellites, the $\mathrm{F} 3 / \mathrm{C}$ ionospheric topside electron densities have been validated (Lai et al. 2013; Pedatella et al. 2015). Results show the good agreement between the F3/C GPS RO and the C/NOFS in-situ observations. However, the C/NOFS satellite was placed into a low Earth orbit with a perigee height of $\sim 400 \mathrm{~km}$ and an apogee of $\sim 850 \mathrm{~km}$, it is 
hard to directly compare its in-situ measurements with the topmost electron densities of the F3/C GPS RO, which is an important parameter for the Abel inversion (Lei et al. 2007).

Following on the F3/C mission, the six FORMOSAT-7/ COSMIC-2 (F7/C2) satellites were launched on 25 June 2019 in a low earth orbit (LEO) with $24^{\circ}$ inclination angle and $\sim 550 \mathrm{~km}$ altitude. All six satellites have been receiving GPS and Global Navigation Satellite System (GLONASS) signals, which providing around 4000 ionospheric EDPs per day between $50^{\circ}$ north and south latitudes. Another on board instrument, call as Ion Velocity Meter (IVM), can measures the in-situ temperature, velocity, and density of ions in the path of each F7/C2 satellite. These in-situ observations provide us a good opportunity to directly evaluate the system errors of the Abel inversion. By employing the in-situ ion densities measured by the IVM experiment on the F7/C2 satellite at the orbit altitude, the main objective of this study is to validate the topmost electron density of F7/C2 RO EDPs.

\section{RESULTS AND DISCUSSIONS}

The topmost electron densities as well as the in-situ ion densities at the orbit altitude on 1 January 2021 have been examined in this study. At the beginning of January 2021, five LEO satellites had been transferred to the $550-\mathrm{km}$ final mission orbits and only one satellite was still on its parking orbit of about $720 \mathrm{~km}$ altitude. Based on the first order estimation of orbit electron density in the Abel inversion (cf. Syndergaard et al. 2006), the different satellite orbits of 550 and $720 \mathrm{~km}$ altitude could significantly influence the retrieved electron density profiles. Furthermore, it is also found that the accuracy of IVM ion density is better at the low orbit $(\sim 540 \mathrm{~km})$ than that at the high orbits $(\sim 710 \mathrm{~km})$ (Wu et al. 2021). Therefore, we only choose both RO and IVM measurements when the satellite arrived to $\sim 550 \mathrm{~km}$ altitude to exclude the orbit influence. Based on the above selection, there are 3965 EDPs obtained. Figure 1 shows the ground projected locations of one EDP (blue line) and the in-situ ion density (red line) during 12:00:48 UT to 12:07:36 UT on 1 January 2021 as an example. This is an ascending pass case, showing the LEO satellite flying from the north-west to the south-east direction. The topmost electron density occurred at 12:07:36 UT and its location is around latitude of $-18.55^{\circ} \mathrm{N}$, longitude of $-42.79^{\circ} \mathrm{E}$, and altitude of $540.22 \mathrm{~km}$, which is very close to the LEO satellite location $\left(-18.01^{\circ} \mathrm{N},-43.52^{\circ} \mathrm{E}, 541.08 \mathrm{~km}\right)$ at 12:07:36 UT.

The 3058 topmost electron densities within $2^{\circ}$ horizontal distance from the LEO satellite were selected for the comparison. Figure 2 presents the comparison between the GPS RO and the IVM ion density observations for the daytime (06 - 18 LT) and the nighttime (18 - 06 LT). It shows that the overall relationship between these two kinds of observations has a strong correlation since the value of correlation coefficient is greater than 0.9 . This result is similar to the previous studies (Lai et al. 2013; Pedatella et al. 2015), which compared the F3/C GPS RO electron densities with the in-situ electron densities from the C/NOFS satellite at its orbital altitude, around 400 to $850 \mathrm{~km}$. Figure 2 is also found that most of the scattered points are concentrated at the end of the line $\mathrm{y}=\mathrm{x}$ during the daytime and concentrated at the beginning of the line $y=x$ during the nighttime. The correlation coefficients for the daytime and the nighttime are 0.89 and 0.86 (not shown), respectively. This difference might come from the relatively small background electron densities during nighttime which has a relatively larger uncertainty of the calibrated TEC around the topside EDPs in the Abel inversion (Yue et al. 2011).

In order to know and evaluate the deviation of topmost electron density from the in-situ ion density, their differences as well as the root-mean-square-difference (RMSD) is computed. The RMSD is defined as

$$
R M S D=\sqrt{\frac{\sum_{i=1}^{N}\left(D_{R O}-D_{I V M}\right)^{2}}{N}}
$$

in which $N$ is the total number of observations (3058). $D$ is either the topmost electron density $\left(D_{R O}\right.$ in the equation) or the in-situ ion density ( $D_{I V M}$ in the equation). Figure 3 presents the residual distribution histogram of their differences for all local times. It shows a mean of residual of $-7.6 \times 10^{3}$ ele $\mathrm{cm}^{-3}$, a standard deviation of $3.6 \times 10^{4} \mathrm{ele}$ $\mathrm{cm}^{-3}$, and a RMSD of $3.6 \times 10^{4}$ ele $\mathrm{cm}^{-3}$, indicating that they match well but the topmost electron density is slightly lower than the in-situ ion density. This might be caused by the estimation of the electron density at the satellite orbit altitude (Lei et al. 2007; Yue et al. 2010, 2011). The orbit electron density is derived from the calibrated TEC below the orbit altitude by fitting a linear regression of square root function under the assumption of spherical symmetry for the electron density (cf. Syndergaard et al. 2006), which results in systematic biases in the standard Abel inversion processes (Schreiner et al. 1999; Lei et al. 2007; Wu et al. 2009). By compared F3/C GPS RO electron density in the topside ionosphere with in-situ electron density from $\mathrm{C} /$ NOFS, Pedatella et al. (2015) further suggested that the error introduced by the Abel inversion spherical symmetry assumption increases with decrease of altitude due to the higher and more structured electron densities at lower altitude. The mission orbit of $\mathrm{F} 7 / \mathrm{C} 2$ satellite is around $550 \mathrm{~km}$, which is lower than that of $\mathrm{F} 3 / \mathrm{C}$ satellite $(\sim 800 \mathrm{~km})$. It can be expected that the spherical symmetry assumption and the square root fitting might be sensitive to the estimation of topmost electron densities at F7/C2 orbit altitudes and the induced errors will be propagated to the bottom layer. If one can retrieve the electron density profiles by employing the in-situ orbit ion density from IVM, the accuracy of EDP approximates might be improved. 
In the standard Abel inversion, there is another assumption that the LEOs and the occultation planes are coplanar. However, in the most situations, they are not exactly coplanar, which indicates that the coplanar assumption might cause the inversion error in the EDP. Lin et al. (2013) developed a technique based on the epoch difference inversion (EDI) to retrieve the EDPs without the coplanar assumption. Their results presented that the EDI has better performance than the standard Abel inversion, showing that the coplanar approximation is important to influence the accuracy of EDP retrieve. In order to quantitatively evaluate the impact of coplanar assumption to the topmost electron density, in this study, we further calculate the dihedral angle of the LEO and the occultation plane. Figure 4 illustrates the geometry of the LEO satellite and the GNSS (Global Navigation Satellite Systems) satellite not to scale. From the normal vectors of the LEO-LEO-Earth plane (blue vector) and the LEO-GNSS-Earth plane (red vector), we can calculate their dihedral angle by the dot product. In the case of Fig. 4, the LEO and the occultation plane is not coplanar with a dihedral angle of $\alpha$. The dihedral angle is less than $90^{\circ}$. We, then, calculate the dihedral angles for each GPS RO and IVM comparison in Fig. 2 and further divide these angles into 6 equal sectors, $15^{\circ}$ each. Figures $5 \mathrm{a}$ and $\mathrm{b}$ show

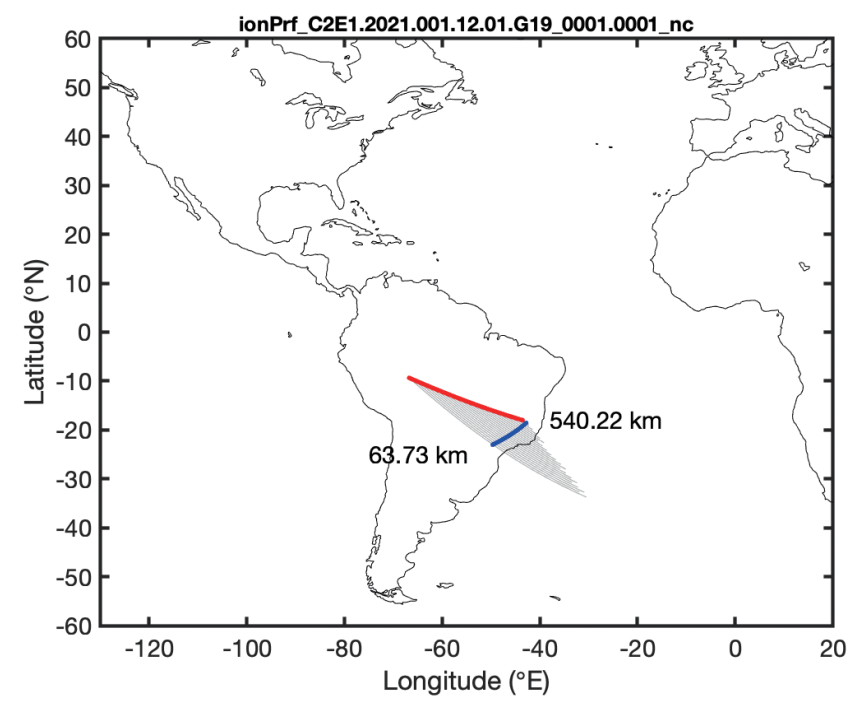

Fig. 1. The ground projected locations of one radio occultation event during 12:00:48 UT to 12:07:36 UT on 1 January 2021. The red and blue dots indicate the locations of IVM in-situ ion density and GPS RO electron density profile, respectively.

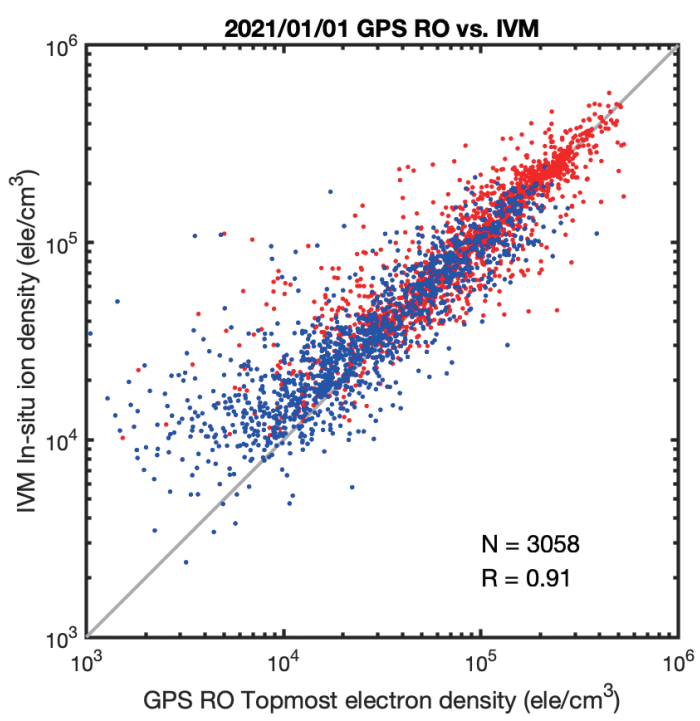

Fig. 2. The comparison between the topmost electron densities from GPS RO EDPs and the in-situ ion density from IVM. The red and blue dots indicate the daytime (06 - 18 LT) and nighttime (18 - 06 LT) observations. The gray line is the line that the IVM equals the GPS RO. The total observation number $(\mathrm{N})$ is 3058 and the correlation coefficient $(\mathrm{R})$ is 0.91 . 


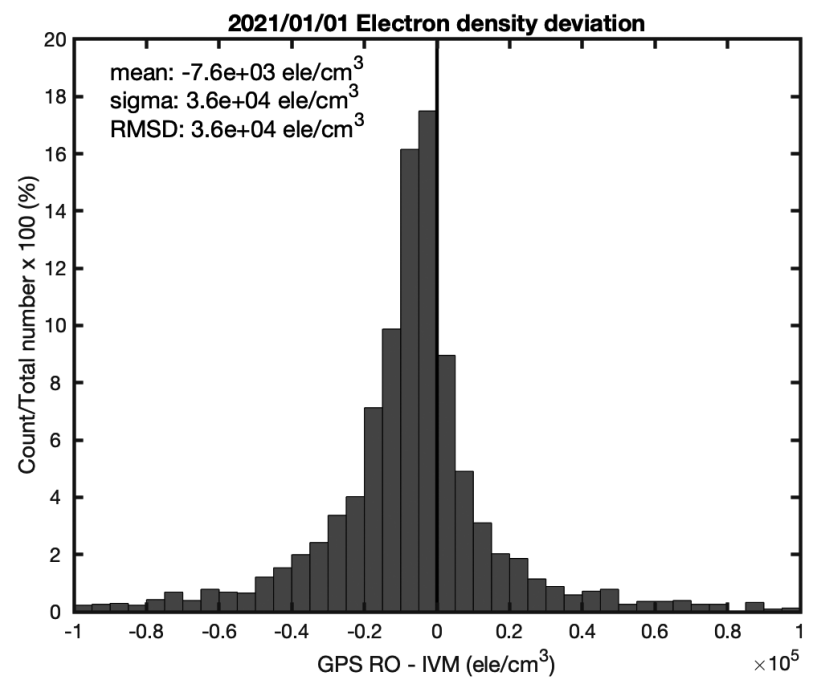

Fig. 3. The histogram of the residual distribution between GPS RO and IVM for all local times. The y-axes value is identified by the count in each residual interval divided by the total observation number (3058).

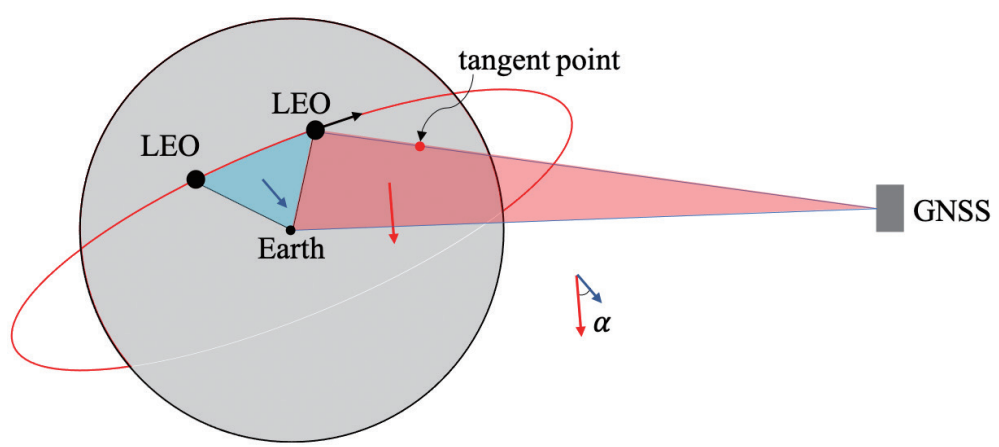

Fig. 4. The sketch of the geometry of the LEO and GNSS satellites. The term of 'Earth' in the sketch indicates the earth's center. The blue and red lines indicate the normal vectors of the LEO-LEO-Earth plane and the LEO-GNSS-Earth plane, respectively.
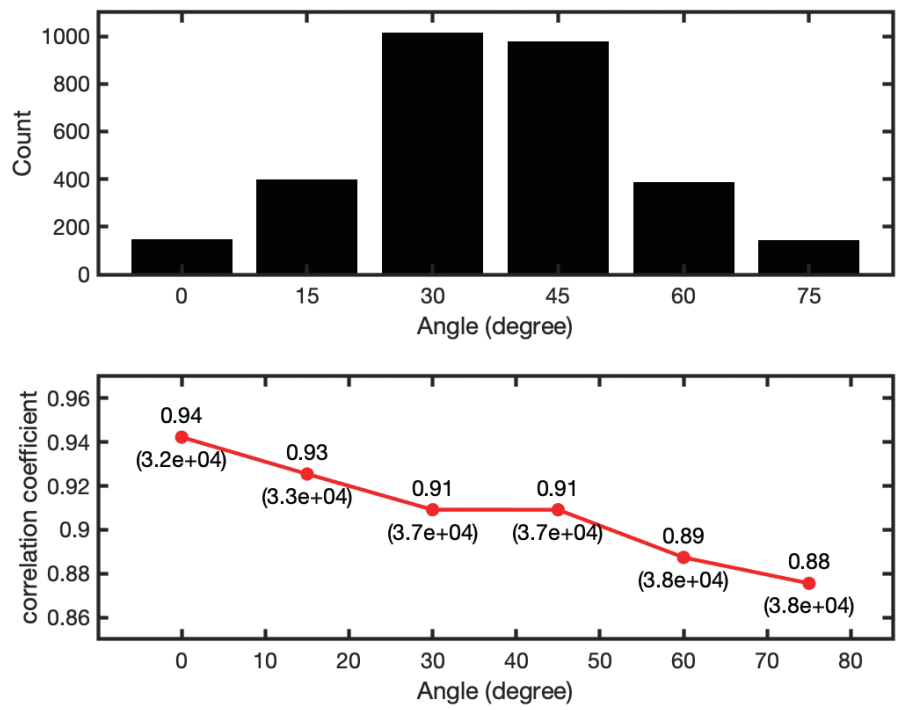

Fig. 5. The observation numbers (a) and the correlation coefficients and RMSDs (b) at different dihedral angle sectors. The angles are divided into 6 equal sectors, $0-15^{\circ}, 15-30^{\circ}, 30-45^{\circ}, 45-60^{\circ}, 60-75^{\circ}$, and $75-90^{\circ}$. The RMSD value is shown by the parentheses in (b). The unit of RMSD is ele $\mathrm{cm}^{-3}$. 
the observation numbers and the correlation coefficients and RMSDs in each sector, respectively. The observation number shows that the most observations are concentrated in the angle of 30 to $60^{\circ}$. It is also clearly seen that the correlation coefficient decreases from 0.94 to 0.88 with the increase of dihedral angle. The RMSDs also show the similar tendency, indicating that the better agreements of topside electron density between the topmost electron density and the in-situ ion density occur at the situation of small dihedral angles. This result is in line with our expected that larger angles lead to more sensitivity to the horizontal density gradient, resulting in the electron density errors on the topside EDP estimation.

\section{CONCLUSION}

This paper firstly evaluates the linear relationship between the estimated topmost electron densities from the F7/ C2 GPS RO EDPs and the collocated in-situ ion density observations from the F7/C2 IVM instrument. The scatter and histogram plots between the topmost electron densities and the in-situ ion densities on 1 January 2021 are employed in this study. The obtained results can be summarized as follows:

(1) The correlation coefficient results reveal the overall good agreement between these two kinds of observations but has a slightly underestimation in the retrieved topmost electron densities. This discrepancy might be attributed to the estimation/assumption of topside electron density at satellite altitudes in the Abel inversion. Applying the in-situ orbit ion densities from F7/C2 IVM to the topmost electron densities with the Abel inversion is expected to improve the accuracy of EDP estimation.

(2) The electron density errors of topmost electron densities increase with increasing dihedral angle between the LEO and the RO planes.

Acknowledgements The study is supported by National Space Organization (NSPO) and Ministry of Science and Technology (MOST) of Taiwan to National Cheng Kung University under NSPO-S-108002, NSPO-S-109059, and MOST-109-2121-M-006-014. The authors would like to thank the Taiwan Analysis Center for COSMIC (TACC) team for providing the RO-derived electron density profiles as well as the in-situ ion densities of FORMOSAT-7/ COSMIC-2 mission at the database (https://tacc.cwb.gov. tw/v2/download.html).

\section{REFERENCE}

Kelley, M. C., V. K. Wong, N. Aponte, C. Coker, A. J. Mannucci, and A. Komjathy, 2009: Comparison of COSMIC occultation-based electron density profiles and TIP observations with Arecibo incoherent scatter radar data. Radio Sci., 44, RS4011, doi:
10.1029/2008RS004087. [Link]

Lai, P.-C., W. J. Burke, and L. C. Gentile, 2013: Topside electron density profiles observed at low latitudes by COSMIC and compared with in situ ion densities measured by C/NOFS. J. Geophys. Res., 118, 2670-2680, doi: 10.1002/jgra.50287. [Link]

Lee, I. T., T. Matsuo, A. D. Richmond, J. Y. Liu, W. Wang, C. H. Lin, J. L. Anderson, and M. Q. Chen, 2012: Assimilation of FORMOSAT-3/COSMIC electron density profiles into a coupled thermosphere/ionosphere model using ensemble Kalman filtering. J. Geophys. Res., 117, A10318, doi: 10.1029/2012JA017700. [Link]

Lei, J., S. Syndergaard, A. G. Burns, S. C. Solomon, W. Wang, Z. Zeng, R. G. Roble, Q. Wu, Y.-H. Kuo, J. M. Holt, S.-R. Zhang, D. L. Hysell, F. S. Rodrigues, and C. H. Lin, 2007: Comparison of COSMIC ionospheric measurements with ground-based observations and model predictions: Preliminary results. J. Geophys. Res., 112, A07308, doi: 10.1029/2006JA012240. [Link]

Lin, C. H., C. H. Liu, J. Y. Liu, C. H. Chen, A. G. Burns, and W. Wang, 2010: Midlatitude summer nighttime anomaly of the ionospheric electron density observed by FORMOSAT-3/COSMIC. J. Geophys. Res., 115, A03308, doi: 10.1029/2009JA014084. [Link]

Lin, C. Y., T. Matsuo, J. Y. Liu, C. H. Lin, J. D. Huba, H. F. Tsai, and C. Y. Chen, 2017: Data assimilation of ground-based GPS and radio occultation total electron content for global ionospheric specification. J. Geophys. Res., 122, 10876-10886, doi: 10.1002/2017JA024185. [Link]

Lin, J., J. Xiong, F. Zhu, J. Yang, and X. Qiao, 2013: An ionospheric occultation inversion technique based on epoch difference. J. Atmos. Sol.-Terr. Phys., 102, 192197, doi: 10.1016/j.jastp.2013.05.003. [Link]

Luan, X., W. Wang, A. Burns, S. C. Solomon, and J. Lei, 2008: Midlatitude nighttime enhancement in $\mathrm{F}$ region electron density from global COSMIC measurements under solar minimum winter condition. J. Geophys. Res., 113, A09319, doi: 10.1029/2008JA013063. [Link]

Pedatella, N. M., X. Yue, and W. S. Schreiner, 2015: Comparison between GPS radio occultation electron densities and in situ satellite observations. Radio Sci., 50, 518-525, doi: 10.1002/2015RS005677. [Link]

Schreiner, W. S., S. V. Sokolovskiy, C. Rocken, and D. C. Hunt, 1999: Analysis and validation of GPS/MET radio occultation data in the ionosphere. Radio Sci., 34, 949-966, doi: 10.1029/1999RS900034. [Link]

Syndergaard, S., W. S. Schreiner, C. Rocken, D. C. Hunt, and K. F. Dymond, 2006: Preparing for COSMIC: Inversion and Analysis of Ionospheric Data Products. In: Foelsche, U., G. Kirchengast, and A. Steiner (Eds.), Atmosphere and Climate: Studies by Occultation 
Methods, Springer Berlin Heidelberg, doi: 10.1007/3540-34121-8_12. [Link]

Wu, Q., N. Pedatella, J. Braun, and M. Chou, 2021: Intercalibration of ionospheric density data from IVM and TGRS. $8^{\text {th }}$ International Radio Occultation Working Group Meeting - IROWG-8, Virtual Meeting, April.

Wu, X., X. Hu, X. Gong, X. Zhang, and X. Wang, 2009: Analysis of inversion errors of ionospheric radio occultation. GPS Solut., 13, 231-239, doi: 10.1007/ s10291-008-0116-x. [Link]
Yue, X., W. S. Schreiner, J. Lei, S. V. Sokolovskiy, C. Rocken, D. C. Hunt, and Y.-H. Kuo, 2010: Error analysis of Abel retrieved electron density profiles from radio occultation measurements. Ann. Geophys., 28, 217-222, doi: 10.5194/angeo-28-217-2010. [Link]

Yue, X., W. S. Schreiner, C. Rocken, and Y.-H. Kuo, 2011: Evaluation of the orbit altitude electron density estimation and its effect on the Abel inversion from radio occultation measurements. Radio Sci., 46, RS1013, doi: 10.1029/2010RS004514. [Link] 